\title{
Mid-infrared observations of the ultraluminous galaxies IRAS 14348-1447, IRAS 19254-7245, and IRAS 23128-5919^
}

\author{
V. Charmandaris ${ }^{1}$, O. Laurent ${ }^{2,3}$, E. Le Floc'h ${ }^{2}$, I. F. Mirabel ${ }^{2,4}$, M. Sauvage ${ }^{2}$, S. C. Madden ${ }^{2}$, P. Gallais ${ }^{2}$, \\ L. Vigroux ${ }^{2}$, and C. J. Cesarsky ${ }^{5}$ \\ 1 Cornell University, Astronomy Department, Ithaca, NY 14853, USA \\ 2 CEA/DSM/DAPNIA Service d'Astrophysique, 91191 Gif-sur-Yvette, France \\ 3 Max Planck Institut für extraterrestrische Physik, PO Box 1312, 85740 Garching, Germany \\ ${ }^{4}$ Instituto de Astronomía y Física del Espacio, Conicet, cc 67, suc 28. 1428 Buenos Aires, Argentina \\ 5 European Southern Observatory, Karl-Schwarzschild-Str, 85748 Garching bei München, Germany
}

Received 1 March 2002 / Accepted 30 May 2002

\begin{abstract}
We present a study of the three ultraluminous infrared galaxies IRAS 14348-1447, IRAS 19254-7245, and IRAS 23128-5919, based on mid-infrared (MIR) spectro-imaging $(5-18 \mu \mathrm{m})$ observations performed with ISOCAM. We find that the MIR emission from each system, which consists of a pair of interacting late type galaxies, is principally confined to the nuclear regions with diameters of $1-2 \mathrm{kpc}$ and can account for more than $95 \%$ of their IRAS $12 \mu \mathrm{m}$ flux. In each interacting system, the galaxy hosting an active galactic nucleus (AGN) dominates the total spectrum and shows stronger dust continuum $(12-16 \mu \mathrm{m})$ relative to the Unidentified Infrared Band (UIB) emission $(6-9 \mu \mathrm{m})$, suggestive of its enhanced radiation field. The MIR dominant galaxy also exhibits elevated $15 \mu \mathrm{m} / \mathrm{H} \alpha$ and $15 \mu \mathrm{m} / \mathrm{K}$ ratios which trace the high extinction due to the large quantities of molecular gas and dust present in its central regions. Using only diagnostics based on our mid-infrared spectra, we can establish that the Seyfert galaxy IRAS 19254-7245 exhibits MIR spectral features of an AGN while the MIR spectrum of the Seyfert (or LINER) member of IRAS 23128-5919 is characteristic of dust emission principally heated by star forming regions.
\end{abstract}

Key words. galaxies: active - galaxies: individual: IRAS 14348-1447 - galaxies: individual: IRAS 19254-7245 galaxies: individual: IRAS 23128-5919 - galaxies: interactions - infrared: galaxies

\section{Introduction}

It is currently widely accepted that the majority of the most luminous galaxies $\left(L_{\mathrm{bol}}>10^{11} L_{\odot}\right)$ in the local universe $(z<0.3)$ are luminous in the infrared, and include the ultraluminous infrared galaxies (ULIRGs, $L_{\mathrm{IR}}>10^{12} L_{\odot}$ ) which emit the bulk of their energy at infrared wavelengths (Houck et al. 1984; Soifer et al. 1989; Sanders \& Mirabel 1996 and references therein). In those systems most of the infrared emission seems to originate from their dusty nuclear regions. Even though one of the principal heating mechanisms for the lowest luminosity $\left(\lesssim 10^{11} L_{\odot}\right)$ infrared galaxies is the stellar radiation field of young massive stars, it is still unclear if the star formation is also the dominant heating source for ULIRGs or whether one needs to invoke an active galactic nucleus (AGN) and its strong radiation field as the central engine responsible for the heating of the

Send offprint requests to: V. Charmandaris,

e-mail: vassilis@astro.cornell.edu

^ Based on observations with ISO, an ESA project with instruments funded by ESA Member States (especially the PI countries: France, Germany, Netherlands and the UK) and with participation of ISAS and NASA. dust (see Joseph 1999; Sanders 1999). The presence of large quantities of molecular gas has long been detected in the central regions of most ULIRGs (e.g. Sanders \& Mirabel 1985; Sanders et al. 1991) leading to high extinction of both their UV and optical radiation. As a result, since it appears that most galaxies do harbor a super-massive, though often quiescent, black hole (Richstone et al. 1998), one would expect to find in their galactic nucleus observational evidence for a mixture of AGN (Sanders et al. 1988) and/or strong compact starburst regions (Condon et al. 1991) fueled by the high concentration of molecular gas (Bryant \& Scoville 1999). Observations in the mid-infrared (MIR), which are less affected by absorption than shorter wavelengths $\left(A_{15 \mu \mathrm{m}} \sim A_{V} / 70\right.$, Mathis 1990), thus provide a powerful probe of galactic central regions (Soifer et al. 2000, 2001).

As we discussed in Laurent et al. (2000), the integrated MIR emission in active galaxies is produced mainly by the interstellar dust which is heated directly by the ionization field from young stars or an AGN. This is in contrast to late type galaxies where the MIR $(5-20 \mu \mathrm{m})$ energy budget is dominated by the reprocessed emission of star forming regions in their disk and accounts for $\sim 15 \%$ of their luminosity 
(Dale et al. 2001; Helou et al. 2001; Roussel et al. 2001). However, the main difficulty in assessing the importance of the underlying physics in galactic nuclei, where the spatial resolution is typically poor, is in separating the contribution of star forming regions and the active nucleus from the integrated MIR emission. The development, application, and general utility of MIR diagnostics in nuclei of galaxies has already been demonstrated by Roche et al. (1991) and more recently by Genzel et al. (1998); Laurent et al. (2000), as well as by Dudley (1999); Imanishi \& Dudley (2000). This was mainly accomplished with the advent of ISOCAM and SWS on board ISO, with high spatial and spectral resolution, as well as improved sensitivity in the 3 to $\sim 40 \mu \mathrm{m}$ wavelength range, thus allowing us to study the nature of the heating sources in ULIRGs. More specifically it has been shown by Lutz et al. (1998), Laurent et al. (1999b), Laurent et al. (2000), Tran et al. (2001) that a nearby galaxy hosting a dominant AGN is clearly different in the MIR from a starburst or a late type spiral. The most striking difference is that the rather featureless MIR spectrum in AGN lacks the emission bands at 6.2, 7.7, 8.6, 11.3 and $12.7 \mu \mathrm{m}$, which are seen in late type galaxies and are attributed to Polycyclic Aromatic Hydrocarbons (PAHs) - also often called Unidentified Infrared Bands (UIBs). One may consider that this is simply due to the fact that its elevated MIR continuum of the AGN overwhelms any UIB feature emission (Pier \& Krolik 1992; Barvainis 1987). It seems inevitable that as the AGN heats its dusty torus at $T \sim 1000 \mathrm{~K}$ and the dust grains approach sublimation temperatures, the more fragile molecules responsibly for the UIB emission could be partly destroyed by a photo-thermo-dissociation mechanism (Leger et al. 1989). Obviously this picture is more complicated in distant galaxies since due to limited spatial resolution the contribution of the star forming regions surrounding an AGN would progressively enter into the beam and dilute any AGN MIR signature (see Laurent et al. 1999b). When sufficient spatial resolution is available to directly view the active nucleus, as is often the case in Seyfert 1 galaxies, the non-thermal emission from the AGN will dominate the spectrum. Consequently, the spectrum can then be fitted by a power law and has a "bump" in the 4-5 $\mu \mathrm{m}$ range. A 5-11 $\mu \mathrm{m}$ study of a large sample of Seyfert galaxies with ISO by Clavel et al. (2000) confirmed this picture, concluding that Seyfert 2 galaxies have weaker MIR continuum. However, a detailed analysis of the MIR spectra and images of the prototypical Seyfert 2 galaxy NGC 1068 by Le Floc'h et al. (2001) showed that if sufficient spatial resolution is available and the AGN is extremely strong, even in the case of a Seyfert 2 one can isolate the emission of the central engine from the star forming regions which surround it. In that case the MIR spectrum of the Seyfert 2 would also be a power law with the addition of a weak PAH emission.

Despite this progress, several questions concerning the extent and spectral characteristics of the MIR emission in active nuclei, as well as the correlation between MIR and optical activity have not been fully examined. Could broad band MIR photometry be used to probe the physical characteristics of AGNs? In the present paper we try to address some of these issues by studying the MIR spectral energy distribution (SED) of three ultraluminous IRAS galaxies. Each IRAS source, the properties of which are presented in Table 1, consists of a merging pair of galaxies with different levels of nuclear activity. The targets were specifically selected as MIR bright and harboring an optically classified AGN. In Sect. 2, we describe the observations and in Sect. 3 we present the details of our study and analysis of the data for each system. A discussion followed by concluding remarks is presented in Sect. 4. Throughout this paper we assume a Hubble constant $H_{0}=75 \mathrm{~km} \mathrm{~s}^{-1} \mathrm{Mpc}^{-1}$ and $q_{0}=1 / 2$.

\section{Observations and data reduction}

Our MIR observations were obtained using ISOCAM, a $32 \times$ 32 pixel array (Cesarsky et al. 1996a) on board the ISO satellite (Kessler et al. 1996). Each system was observed with broad band filters ranging from 5 to $18 \mu \mathrm{m}$ in a $2 \times 2$ raster with 6 pixel offsets and a lens producing a pixel field of view (PFOV) of $1.5^{\prime \prime}$, resulting in a final image of $57^{\prime \prime} \times 57^{\prime \prime}$. This enabled us to obtain images with a spatial resolution of $3^{\prime \prime}$ (at $6 \mu \mathrm{m}$ ) to $4.5^{\prime \prime}(15 \mu \mathrm{m})$ limited by the pixel size at $6 \mu \mathrm{m}$ and by the full width at half maximum (FWHM) of the point spread function (PSF) at $15 \mu \mathrm{m}$. We note the ISOCAM filters by their name and central wavelength. The wavelength range in $\mu \mathrm{m}$ covered by each filter was: $L W 2$ (5.0-8.5), $L W 3$ (12.0-18.0), $L W 4$ (5.56.5), LW6 (7.0-8.5), LW7 (8.5-10.7), LW8 (10.7-12.0), LW9 $(14.0-16.0)$. At subsequent sections in this paper we will refer to the measured flux densities using the various filters as $f_{x \mu \mathrm{m}}$ where $x$ is the central wavelength of each filter in microns.

Spectrophotometric observations were also obtained with the circular variable filter (CVF) for IRAS 23128-5919, the brightest of our sources. The CVF covers a spectral range from 5 to $16.5 \mu \mathrm{m}$ with a $1.5^{\prime \prime}$ PFOV and a spectral resolution of 50 . Each integration step was composed of 12 images with $5.04 \mathrm{~s}$ integration time and during the CVF scan the wavelength step varied between 0.05 and $1.11 \mu \mathrm{m}$. Details on the observing parameters are summarized in Table 2.

The data were analyzed with the CAM Interactive Analysis software $\left(\mathrm{CIA}^{1}\right)$. A dark model taking into account the observing time parameters was subtracted. Cosmic ray contamination was removed by applying a wavelet transform method (Starck et al. 1997). Corrections of detector memory effects were done applying the Fouks-Schubert's method (Coulais \& Abergel 2000). The flat field correction was performed using the library of calibration data. Finally, individual exposures were combined using shift techniques in order to correct the effect of jittering due to the satellite motions (amplitude $\sim 1^{\prime \prime}$ ). A deconvolution using multiscale resolution techniques (Starck et al. 1999) was subsequently applied to estimate the physical size of the quasi-point like sources responsible for the infrared emission in our data (see Sect. 3.1).

The details of the analysis of the ISOPHOT-S data of the three galaxies, which we also include in this paper for reasons of comparison, are published by Rigopoulou et al. (1999).

Based on three different observations of IRAS 19254-7245 taken with identical $L W$ filters but with different roll angle,

${ }^{1}$ CIA is a joint development by the ESA astrophysics division and the ISOCAM consortium. 
integration times per exposure ( $2 \mathrm{~s}$ and $5 \mathrm{~s}$ ) and PFOVs, as well as on similar analysis of other ISOCAM-CVF and ISPHOT-S observations, we estimate that the uncertainty of our photometry measurements is $\sim 20 \%$ (see Table 3 ).

\section{Results}

\subsection{Background and general properties}

The sensitivity and spatial resolution capabilities of ISOCAM enable us to obtain deep maps of the MIR emission of each galaxy. Since the interacting members of the IRAS galaxies are very close and are point-like objects with one member typically dominating the MIR emission, photometry measurements were treated with extra care. Our approach was to fit the MIR PSF of the brightest component and to subtract its contribution from the location of the neighboring, fainter galaxy. We then performed aperture photometry on the fainter component, using an aperture $\sim 4.5^{\prime \prime} \times 4.5^{\prime \prime}$. In spite of the difference in their peak intensities, the relative positions of the nuclei were very well known from deep near-IR imaging (Duc et al. 1997b). Final aperture correction was applied to the flux of each galaxy to account for the overall extension of the PSF. Our measurements are presented in Table 3. We also include the equivalent broad-band filter fluxes estimated from the ISOPHOT-S spectra, which are found in good agreement with our data within the photometric uncertainties. Since the galaxies were observed several times under different ISOCAM configurations, more than one value is often quoted for the same filter. This was done in order to display the internal consistency of the different measurements and their median value should be considered as the nominal flux density of each galaxy.

ISOCAM has detected nearly $\sim 100 \%$ of the $12 \mu \mathrm{m}$ IRAS flux (see Table 1) of these galaxies. Moreover, as it can be seen from the images of the galaxies presented later in this section, no extended extra-nuclear emission, has been detected in any of the galaxies in the MIR. In all cases, the bulk of the flux coming from these objects originates from a region less than $3-4.5^{\prime \prime}$ in diameter (which corresponds to the FWHM of PSFs) associated with the nuclei of the interacting galaxies.

As it has been discussed in several papers describing ISO observations (i.e. Laurent et al. 2000 and references therein) the MIR emission of spiral galaxies observed by ISOCAM originates from a number of physical processes, with two dust heating mechanisms typically prevailing. One is the thermal emission produced by thermally-fluctuating, small grains $(\sim 10 \mathrm{~nm})$ heated by the interstellar radiation field, observed between $12 \mu \mathrm{m}$ and $18 \mu \mathrm{m}$ in areas of strong radiation environments and is often sampled by the $L W 3$ filter. The second is due to the UIBs, which originated from complex 2-dimensional aromatic molecules having $\mathrm{C}=\mathrm{C}$ and $\mathrm{C}-\mathrm{H}$ bonds and can be seen at 6.2, $7.7,8.6,11.3$ and $12.7 \mu \mathrm{m}$ in the ISOCAM wavelength range. The emission in these bands can be observed either with the CVF or using a sequence of narrow-band filters. An absorption feature due to silicates is often observed at $9.7 \mu \mathrm{m}$ and can be measured using the $L W 7(8.5-10.7 \mu \mathrm{m})$ filter. Finally, two forbidden emission lines due to [NeII] at $12.8 \mu \mathrm{m}$ and [NeIII] at $15.5 \mu \mathrm{m}$ can be detected in the CVF mode. A contribution to the MIR spectrum by a third component, the Rayleigh-Jeans tail of an old stellar population, is generally negligible in late type galaxies where the hot dust emission dominates. This MIR emission directly arising from stellar photosphere is detected in early type galaxies (Madden et al. 1997).

Analysis of a wealth of ISOCAM data has shown that the flux ratio of the broad band filters centered at $15 \mu \mathrm{m}$ and $6.75 \mu \mathrm{m}\left(L W 3 / L W 2\right.$ or $\left.f_{15 \mu \mathrm{m}} / f_{6.7 \mu \mathrm{m}}\right)$ provides a diagnostic of the dominant global MIR emission characteristic of $\mathrm{H}_{\text {II }}$ regions, the diffuse interstellar medium or photodissociation regions (Verstraete et al. 1996; Cesarsky et al. 1996b; Dale et al. 2001; Roussel et al. 2001). It has been shown that while quiescent star forming regions typically have $f_{15 \mu \mathrm{m}} / f_{6.7 \mu \mathrm{m}} \sim 1$, in active sites of massive star formation this ratio increases due to the increasing contribution of the continuum emission in the $15 \mu \mathrm{m}$ bandpass (Sauvage et al. 1996; Mirabel et al. 1998; Vigroux et al. 1999; Dale et al. 2001). However, one should note that the use of this indicator alone is not sufficient to distinguish between the MIR spectrum due to star formation or an AGN, since in AGNs the hot dust continuum arising from the torus also has $f_{15 \mu \mathrm{m}} / f_{6.7 \mu \mathrm{m}}>1$. Such a degeneracy may be resolved using the flux ratio of the $6.75 \mu \mathrm{m} \mathrm{LW2}$ filter (sampling the 6.2 and $7.7 \mu \mathrm{m}$ UIBs) to the narrower $L W 4$ filter which is centered at $6.0 \mu \mathrm{m}$ only contains the $6.2 \mu \mathrm{m}$ UIB. As the continuum variation between these two filters is negligible, the $f_{6.7 \mu \mathrm{m}} / f_{6 \mu \mathrm{m}}$ $(L W 2 / L W 4)$ ratio estimates the intensity of UIBs relative to the underlying continuum (see Fig. 5 of Laurent et al. 2000). The closer $f_{6.7 \mu \mathrm{m}} / f_{6 \mu \mathrm{m}}$ is to 1 , the stronger the continuum is. Since AGNs have weaker UIBs than starbursts, Laurent et al. (2000) proposed to use the combination of the $f_{15 \mu \mathrm{m}} / f_{6.7 \mu \mathrm{m}}$ and $f_{6.7 \mu \mathrm{m}} / f_{6 \mu \mathrm{m}}$ colors to differentiate between the two mechanisms contributing to the MIR emission. Clearly there is a redshift dependence of this diagnostic due to the $K$-correction of the SEDs, but since the redshifts of our targets are small, these indicators can be applied (Laurent 1999a).

Using a large sample of galaxies in the Virgo cluster Boselli et al. (1997) studied the properties of their MIR emission, normalized to the mass of these galaxies. This was done by examining the ratios of the $f_{6.7 \mu \mathrm{m}}(L W 2)$ and $f_{15 \mu \mathrm{m}}(L W 3)$ flux densities to the $K$ band light, which scales with stellar mass of the galaxy, and it was found that the typical $f_{15} \mu \mathrm{m} / K$ ratio for a late type spiral ranges between 1 and 10 . In Table 4, we present those ratios for our sample and we find that even though their active nuclei must contribute some non-thermal emission in the $K$ band the ratios are considerably larger. This can be attributed to a combination of increased thermal dust emission along with a wavelength dependent absorption, which, in highly obscured sources, may decrease their $K$ band flux. Such an example is Arp 220 which displays a ratios $f_{15} \mu \mathrm{m} / K \sim 30$ (Charmandaris et al. 2002). Two more ratios of the $L W 3$ and $L W 2$ over the $\mathrm{H} \alpha$ line flux density are also included in Table 4 for reasons of completeness. It has been established that in normal spirals, both filters mostly trace the MIR flux arising from the reprocessing of ionising radiation which is observed in the optical via the $\mathrm{H} \alpha$ line (Sauvage et al. 1996; Roussel et al. 2001; Dale et al. 2001). Since in more active galaxies, the $\mathrm{H} \alpha$ emission is strongly affected by the absorption, these ratios could be used 
Table 1. Properties of the three IRAS systems.

\begin{tabular}{|c|c|c|c|c|c|c|c|c|c|c|}
\hline \multirow{2}{*}{$\begin{array}{c}\text { Target } \\
\text { IRAS Name }\end{array}$} & \multirow{2}{*}{$\begin{array}{c}\text { RA } \\
\text { J2000.0 }\end{array}$} & \multirow{2}{*}{$\begin{array}{c}\text { DEC } \\
\mathrm{J} 2000.0\end{array}$} & \multirow[t]{2}{*}{$z$} & \multicolumn{4}{|c|}{$F_{v}(\mathrm{Jy})$} & \multirow{2}{*}{$\begin{array}{c}D_{\mathrm{L}} \\
(\mathrm{Mpc})\end{array}$} & \multirow{2}{*}{$\begin{array}{c}\log \left(L_{\mathrm{FIR}}\right) \\
\left(L_{\odot}\right)\end{array}$} & \multirow{2}{*}{$\begin{array}{c}\log \left(L_{\mathrm{IR}}\right) \\
\left(L_{\odot}\right)\end{array}$} \\
\hline & & & & $12 \mu \mathrm{m}$ & $25 \mu \mathrm{m}$ & $60 \mu \mathrm{m}$ & $100 \mu \mathrm{m}$ & & & \\
\hline $19254-7245$ & $19.31^{\mathrm{m}} \cdot 21.6^{\mathrm{s}}$. & $-72^{\circ} .39 .20 .8^{\prime \prime}$ & 0.0617 & 0.22 & 1.24 & 5.48 & 5.79 & 250 & 11.68 & 12.01 \\
\hline 23128-5919 & $23^{\mathrm{h}} \cdot 15^{\mathrm{m}} \cdot 46.9$. & $-59^{\circ} .03{ }^{\prime} 14.2^{\prime \prime}$ & 0.0446 & 0.24 & 1.59 & 10.80 & 10.99 & 180 & 11.69 & 11.96 \\
\hline $14348-1447$ & $14^{\mathrm{h}} 37^{\mathrm{m}} \cdot 38.2^{\mathrm{s}}$. & $-15^{\circ} .00^{\prime} 23.9^{\prime}$ & 0.0823 & $<0.14$ & 0.49 & 6.87 & 7.07 & 335 & 12.05 & 12.27 \\
\hline
\end{tabular}

Table note: The far-infrared and infrared luminosities are calculated using $L_{\mathrm{FIR}}=3.94 \times 10^{5} \times D(\mathrm{Mpc})^{2}\left(2.58 \times f_{60}+f_{100}\right)$ and $L_{\mathrm{IR}}=5.62 \times$ $10^{5} \times D(\mathrm{Mpc})^{2}\left(13.48 \times f_{12}+5.16 \times f_{25}+2.58 \times f_{60}+f_{100}\right)$ respectively, where the luminosity distance is defined as $D_{\mathrm{L}}=\frac{c}{H_{0} q_{0}^{2}}\left(z q_{0}+\left(q_{0}-\right.\right.$ $1)\left(\sqrt{\left(1+2 q_{0} z\right)}-1\right)($ see Sanders \& Mirabel 1996).

Table 2. ISOCAM observing parameters.

\begin{tabular}{|c|c|c|c|c|c|c|c|c|c|}
\hline Target & $\begin{array}{l}\text { ISOCAM Filter: } \\
\text { Filter Center: }\end{array}$ & $\begin{array}{c}L W 2 \\
6.75 \mu \mathrm{m}\end{array}$ & $\begin{array}{c}L W 3 \\
15 \mu \mathrm{m}\end{array}$ & $\begin{array}{l}L W 4 \\
6 \mu \mathrm{m}\end{array}$ & $\begin{array}{c}L W 6 \\
7.75 \mu \mathrm{m}\end{array}$ & $\begin{array}{c}L W 7 \\
9.62 \mu \mathrm{m}\end{array}$ & $\begin{array}{c}L W 8 \\
11.4 \mu \mathrm{m}\end{array}$ & $\begin{array}{c}L W 9 \\
15 \mu \mathrm{m}\end{array}$ & $\begin{array}{c}\mathrm{CVF} \\
-\end{array}$ \\
\hline IRAS $19254-7245^{1}$ & & 15.3 & 15.3 & 15.4 & 15.2 & 15.4 & 15.3 & 15.4 & - \\
\hline IRAS $19254-7245^{2}$ & & 7.1 & 7.0 & 11.3 & - & 8.3 & - & - & - \\
\hline IRAS $19254-7245^{3}$ & & 3.4 & 3.6 & - & - & - & - & - & - \\
\hline IRAS $23128-5919^{4}$ & & 7.2 & 7.0 & 11.5 & - & 8.5 & - & - & - \\
\hline IRAS $23128-5919^{5}$ & & - & - & - & - & - & - & - & 148.7 \\
\hline IRAS $14348-1447^{6}$ & & 8.6 & 8.4 & - & - & - & - & - & - \\
\hline
\end{tabular}

Table note: The numbers following each galaxy denote the total on-source exposure time (in minutes) for each filter used, and two galaxies were observed more than once under different configurations, the details of which are: (1) IRAS 19254-7245 observed in proposal CAMACTI2 (PI I.F. Mirabel), $7 L W$ filters, integration time per frame Tint $=5 \mathrm{~s}$, pfov $=1.5^{\prime \prime}$. (2) IRAS19254-7245 observed in proposal CAMACTIV (PI I.F. Mirabel), $4 L W$ filters, Tint $=2 \mathrm{~s}$, pfov $=1.5^{\prime \prime}$. (3) IRAS19254-7245 observed in proposal SAM12N_2 proposal (PI L. Spinoglio), $2 L W$ filters, Tint $=2 \mathrm{~s}$, pfov $=3^{\prime \prime}$. (4) IRAS 23128-5919 observed in proposal CAMACTIV (PI I.F. Mirabel), 4 LW filters, Tint $=2 \mathrm{~s}$, pfov $=1.5^{\prime \prime}$. (5) IRAS 23128-5919 observed in proposal CAMACTI2 (PI I.F. Mirabel), CVF, Tint $=5 \mathrm{~s}$, pfov =1.5". (6) IRAS 14348-1447 observed in proposal CAMACTIV (PI I.F. Mirabel), $2 L W$ filters, Tint $=2 \mathrm{~s}$, pfov $=1.5^{\prime \prime}$.

to quantify the level of absorption ${ }^{2}$ even though one should be cautious in their quantitative interpretation since the ratios may saturate toward extreme starbursts (Roussel et al. 2001). We present the $L W 2 / \mathrm{H} \alpha$ mainly for comparison, as the most interesting indicator is clearly the one involving the $L W 3$ filter which directly traces the continuum of hot dust emission emitted by the small grains.

Finally, in Table 5 we also present the MIR luminosities of both the $L W 2$ and $L W 3$ filters for each galaxy of our sample. One can clearly see that despite the activity in these systems, the MIR spectrum contains only a small fraction $(<5 \%)$ of their energy which is mostly emitted at longer wavelengths in the far-infrared (FIR). This is in sharp contrast from what is seen in normal late type galaxies where $\sim 15 \%$ of the luminosity is emitted between 5-20 $\mu \mathrm{m}$ (Dale et al. 2001). In the same table we include the $L_{\mathrm{IR}}\left(L_{\odot}\right) / M_{\mathrm{H}_{2}}\left(M_{\odot}\right)$ ratio which traces the efficiency of molecular gas consumption, via either star formation or AGN activity, as well as the production of high energy photons which in-turn are reprocessed into infrared via dust absorption and/or scattering. As expected the reported values for our sample are typical of ultraluminous galaxies. Normal spiral galaxies such as the Milky Way have a ratio of $1-10 L_{\odot} M_{\odot}{ }^{-1}$, while starbursts such as M 82 display higher $\sim 100 L_{\odot} M_{\odot}{ }^{-1}$ values (see Sanders et al. 1986; Wild et al. 1992).

\footnotetext{
${ }^{2}$ For a typical visual absorption range of $1-3 \mathrm{mag}, f_{15 \mu \mathrm{m}} / \mathrm{H} \alpha$ varies between 10 and 80 (see Sauvage et al. 1996; Roussel et al. 2001).
}

Let us now review the MIR properties of each system in detail.

\subsection{IRAS 19254-7245}

The ultraluminous infrared galaxy IRAS 19254-7245, also known as the "Superantennae" is the result of a collision between two gas-rich spiral galaxies separated by $10 \mathrm{kpc}\left(8.5^{\prime \prime}\right)$ in projection and displays extremely long tidal tails extending to $350 \mathrm{kpc}$ (Mirabel et al. 1990). Only the MIR emission originating from the nuclear regions of the galaxies is detected in our images (Fig. 1), and there is no evidence for emission extending toward the direction of the tails. Even the northern nucleus is marginally above the sensitivity limit $\sim 1 \mathrm{mJy}$ at $3 \sigma$ (see Table 3).

Using optical spectroscopy, the southern galaxy has been classified as a Seyfert 2 with an observed $F W H M$ of $\sim 1700 \mathrm{~km} \mathrm{~s}^{-1}$ in both permitted and forbidden lines (Mirabel et al. 1990; Duc \& Mirabel 1997a). The presence of an active nucleus is further suggested by the IRAS criteria for selecting Seyferts, since the ratio of its $25 \mu \mathrm{m}$ to the $60 \mu \mathrm{m}$ IRAS flux density is greater than 0.2 (see de Grijp et al. 1985), while its optical and near-infrared colors indicate a strong contribution from a non-thermal component, likely originating from an AGN, as well as emission from very hot dust ( $1000 \mathrm{~K})$ (Vanzi et al. 2002). Evidence of massive star formation is also seen in the nuclear regions as emission line splitting which has been 
Table 3. ISOCAM mid-infrared photometry of the sample.

\begin{tabular}{|c|c|c|c|c|c|c|c|c|c|}
\hline $\begin{array}{l}\text { Target } \\
\text { IRAS }\end{array}$ & $\begin{array}{l}L W 2 \\
(\mathrm{mJy})\end{array}$ & $\begin{array}{l}L W 3 \\
\text { (mJy) }\end{array}$ & $\begin{array}{l}L W 4 \\
(\mathrm{mJy})\end{array}$ & $\begin{array}{l}L W 6 \\
(\mathrm{mJy})\end{array}$ & $\begin{array}{l}L W 7 \\
(\mathrm{mJy})\end{array}$ & $\begin{array}{l}L W 8 \\
(\mathrm{mJy})\end{array}$ & $\begin{array}{l}L W 9 \\
(\mathrm{mJy})\end{array}$ & $\frac{L W 3}{L W 2}$ & $\frac{L W 2}{L W 4}$ \\
\hline $19254 \mathrm{~S}^{1}$ & $106.9 \pm 10.7$ & $284.0 \pm 28.4$ & $90.0 \pm 9.1$ & $150.1 \pm 15.0$ & $91.2 \pm 9.1$ & $107.5 \pm 10.8$ & $337.5 \pm 33.8$ & $2.7 \pm 0.4$ & $1.2 \pm 0.2$ \\
\hline $19254 S^{2}$ & $103.6 \pm 11.0$ & $278.9 \pm 29.1$ & $87.3 \pm 11.2$ & - & $97.1 \pm 10.5$ & - & - & $2.7 \pm 0.4$ & $1.2 \pm 0.2$ \\
\hline $19254 N^{1}$ & $4.8 \pm 0.5$ & $5.9 \pm 0.7$ & $1.9 \pm 0.4$ & $8.3 \pm 0.9$ & $5.1 \pm 0.6$ & $5.9 \pm 0.7$ & $5.4 \pm 1.0$ & $1.2 \pm 0.2$ & $2.5 \pm 0.6$ \\
\hline $19254 N^{2}$ & $3.1 \pm 1.0$ & $7.5 \pm 2.4$ & $1.5 \pm 2.6$ & - & $4.5 \pm 1.7$ & - & - & $2.4 \pm 1.1$ & $2.1 \pm 3.6$ \\
\hline $19254^{1}$ & $111.7 \pm 11.2$ & $289.9 \pm 29.0$ & $91.9 \pm 9.2$ & $158.4 \pm 15.9$ & $96.3 \pm 9.6$ & $113.4 \pm 11.3$ & $342.9 \pm 34.3$ & $2.6 \pm 0.4$ & $1.2 \pm 0.2$ \\
\hline $19254^{2}$ & $106.7 \pm 11.0$ & $286.4 \pm 29.2$ & $88.8 \pm 11.5$ & - & $97.1 \pm 10.6$ & - & - & $2.7 \pm 0.4$ & $1.2 \pm 0.2$ \\
\hline $19254^{3}$ & $114.8 \pm 12.5$ & $290.2 \pm 11.5$ & - & - & - & - & - & $2.5 \pm 0.4$ & - \\
\hline $19254^{\dagger}$ & $113.0 \pm 2.8$ & - & $85.6 \pm 3.7$ & $135.7 \pm 4.1$ & $110.4 \pm 4.6$ & $116.9 \pm 13.9$ & - & - & $1.3 \pm 0.1$ \\
\hline $23128 \mathrm{~S}^{4}$ & $70.8 \pm 7.1$ & $228.3 \pm 22.9$ & $48.5 \pm 5.0$ & - & $67.3 \pm 6.8$ & - & - & $3.2 \pm 0.5$ & $1.5 \pm 0.2$ \\
\hline $23128 \mathrm{~S}^{5}$ & $77.5 \pm 1.6$ & $262.3 \pm 4.3$ & $50.6 \pm 2.3$ & $116.0 \pm 2.6$ & $79.9 \pm 2.0$ & $106.2 \pm 3.6$ & $277.1 \pm 6.6$ & $3.4 \pm 0.1$ & $1.5 \pm 0.1$ \\
\hline $23128 \mathrm{~N}^{4}$ & $38.6 \pm 3.9$ & $88.5 \pm 9.1$ & $19.8 \pm 2.2$ & - & $26.7 \pm 2.8$ & - & - & $2.3 \pm 0.3$ & $2.0 \pm 0.3$ \\
\hline $23128 N^{5}$ & $34.8 \pm 1.2$ & $90.4 \pm 2.1$ & $19.9 \pm 2.0$ & $53.4 \pm 1.7$ & $34.7 \pm 1.5$ & $51.3 \pm 2.4$ & $90.9 \pm 3.2$ & $2.6 \pm 0.1$ & $1.7 \pm 0.2$ \\
\hline $23128^{4}$ & $109.4 \pm 11.0$ & $316.8 \pm 31.7$ & $68.3 \pm 7.0$ & - & $94.0 \pm 9.5$ & - & - & $2.9 \pm 0.4$ & $1.6 \pm 0.2$ \\
\hline $23128^{5}$ & $112.3 \pm 2.6$ & $352.7 \pm 7.2$ & $70.5 \pm 3.4$ & $169.4 \pm 4.4$ & $114.5 \pm 3.1$ & $157.5 \pm 6.1$ & $368.0 \pm 10.7$ & $3.1 \pm 0.1$ & $1.6 \pm 0.1$ \\
\hline $23128^{\dagger}$ & $123.6 \pm 3.2$ & - & $70.0 \pm 3.6$ & $163.0 \pm 4.9$ & $134.5 \pm 4.8$ & $140.4 \pm 14.2$ & - & - & $1.8 \pm 0.1$ \\
\hline $14348 S^{6}$ & $21.8 \pm 4.6$ & $73.9 \pm 9.0$ & - & - & - & - & - & $3.4 \pm 0.8$ & - \\
\hline $14348 N^{6}$ & $11.3 \pm 3.6$ & $22.9 \pm 5.0$ & - & - & - & - & - & $2.0 \pm 0.8$ & - \\
\hline $14348^{6}$ & $33.1 \pm 5.8$ & $96.8 \pm 10.3$ & - & - & - & - & - & $2.9 \pm 0.6$ & - \\
\hline $14348^{\dagger}$ & $37.9 \pm 1.6$ & - & $16.9 \pm 2.0$ & $52.6 \pm 2.4$ & $34.4 \pm 3.3$ & $14.1 \pm 10.7$ & - & - & $2.2 \pm 0.3$ \\
\hline
\end{tabular}

Table note: For each interacting system, we have measured the integrated flux of the individual galaxies resolved by ISOCAM and marked the southern and the northern galaxies with $(\mathrm{S})$ and $(\mathrm{N})$ respectively. We used the same notations as in Table 2 for identifying the different sets of ISOCAM observations, labeled (1) through (6). As all three galaxies were also observed with ISOPHOT-S and one with the CVF, we also provide the equivalent broad-band filter flux estimates (using the known filter band-passes and transmission curves) based on those spectra marked with a $\dagger$ for ISOPHOT-S and a (5) for the CVF. The errors given for each measurement are statistical derived by adding the $1 \sigma$ rms map to the systematic error of $10 \%$ commonly associated with the transient correction. Absolute flux uncertainties are estimated to be $\pm 20 \%$. For the cases where we present multiple measurements for a target their median value should be considered as its nominal flux density.

attributed to a biconical outflow (Colina et al. 1991). The kinetic energy necessary for this to occur can only be produced by supernova explosions or stellar winds further suggesting high star formation rates $\left(150 M_{\odot} \mathrm{yr}^{-1}\right.$, Colina et al. 1991). Ground-based MIR observations at $10 \mu \mathrm{m}$ show that more than $80 \%$ of the total flux originates from the Seyfert 2 (the southern galaxy). The spectrum of the northern galaxy has much weaker emission lines in $\mathrm{H} \alpha$ and [NII], typical of a starburst or LINER (Colina et al. 1991). More recently HST imaging provided new evidence that a double nucleus may be present in both the north and southern components of the Superantennae (Borne et al. 1999), suggesting a multiple merger origin of the system.

Based on the photometry of Table 3, we present in Figs. 2 and 3 the MIR spectral energy distribution for each galaxy, while the integrated MIR SED of the whole system is shown in Fig. 4. In the latter we also compare our data with the spectrum obtained with ISOPHOT-S, the beam of which spatially covered the full emission of IRAS 19254-7245. The extreme difference in the MIR intensities between the southern and northern members are apparant as well as the constrasts in their spectral shape.
Table 4. Broad band color ratios. $\mathrm{H} \alpha$ and $K$ band fluxes are from Duc \& Mirabel (1997a) except the $\mathrm{H} \alpha$ flux of IRAS 14348-1447 (see Veilleux et al. 1995). $L W 2, L W 3$ and $K$ are in mJy and $\mathrm{H} \alpha$ in $10^{-13} \mathrm{erg} \mathrm{cm}^{-2} \mathrm{~s}^{-1}$.

\begin{tabular}{lcccc}
\hline \hline Target & $\frac{L W 2}{\mathrm{H} \alpha}$ & $\frac{L W 2}{K}$ & $\frac{L W 3}{\mathrm{H} \alpha}$ & $\frac{L W 3}{K}$ \\
\hline IRAS 19254-7245(S) & 91.3 & 12.4 & 224.5 & 30.4 \\
IRAS 19254-7245(N) & 78.4 & 1.2 & 85.4 & 1.3 \\
& & & & \\
IRAS 23128-5919(S) & 63.5 & 9.9 & 283.8 & 44.3 \\
IRAS 23128-5919(N) & 31.2 & 8.2 & 85.2 & 22.5 \\
& & & & \\
IRAS 14348-1447(S) & 125.7 & 8.1 & 332.9 & 21.5 \\
IRAS 14348-1447(N) & 161.2 & 6.3 & 263.3 & 10.3 \\
\hline
\end{tabular}

More than $95 \%$ of the MIR emission of IRAS 19254-7245 originates from the southern Seyfert 2 galaxy which displays a peculiar spectrum with a dominant thermal emission at $15 \mu \mathrm{m}$ $\left(f_{15 \mu \mathrm{m}} / f_{6.7 \mu \mathrm{m}} \sim 2.7\right)$ and weak UIBs $\left(f_{6.7 \mu \mathrm{m}} / f_{6 \mu \mathrm{m}} \sim 1.2\right)$. This strong continuum relative to the UIB emission can be the consequence of a high radiation field density mainly produced 

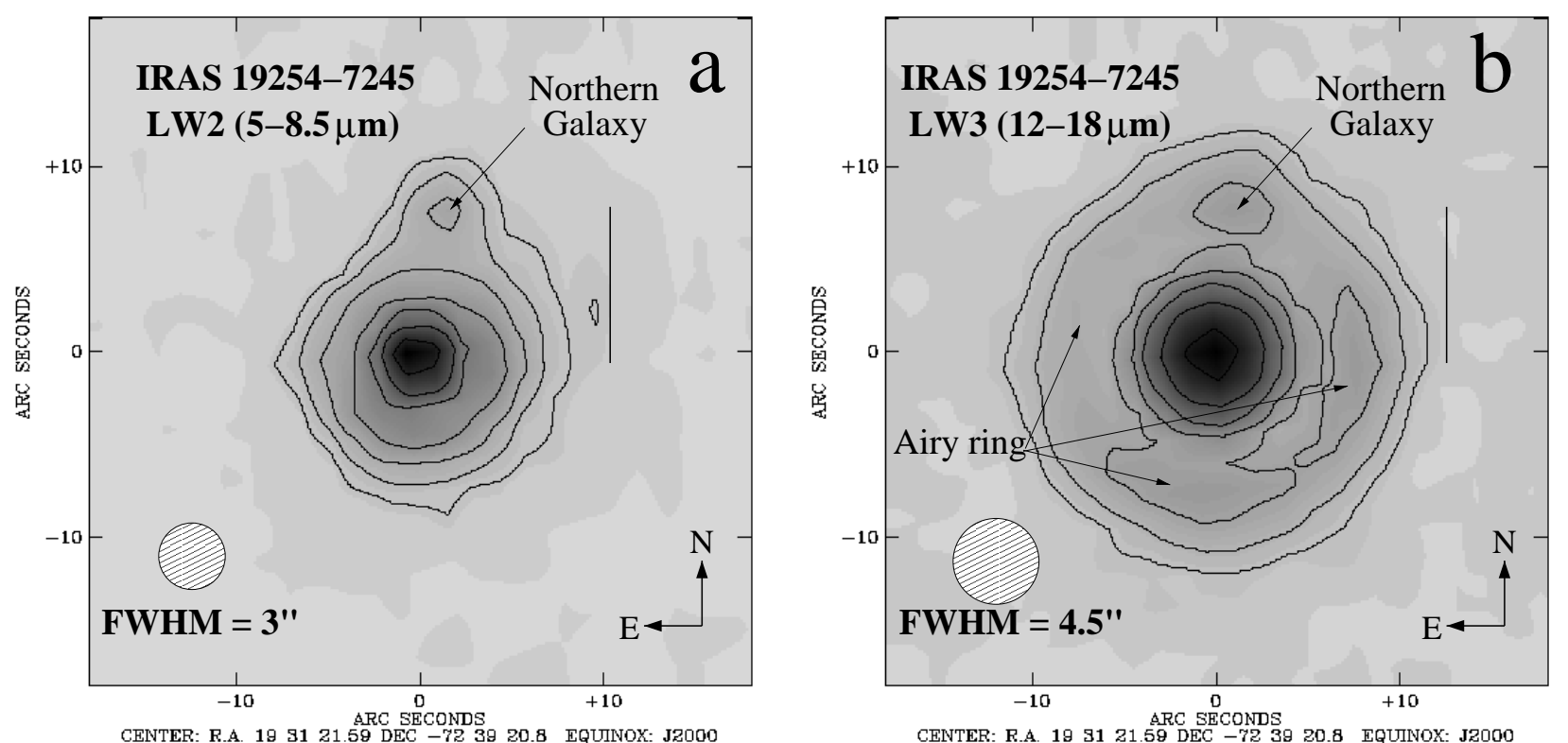

Fig. 1. a) Image of IRAS 19254-7245 observed with ISOCAM at $6.75 \mu \mathrm{m}$ ( $L W 2$ filter). The contours are 5, 10, 20, 40, 80, 160 and $320 \sigma$ $\left(\sigma=0.027 \mathrm{mJy}_{\mathrm{pixel}}{ }^{-1}\right)$. b) Image of IRAS 19254-7245 observed with ISOCAM at $15 \mu \mathrm{m}$ ( $L W 3$ filter). The contours are 5, 10, 20, 40, 80, 160 and $320 \sigma\left(\sigma=0.041 \mathrm{mJy}_{\text {pixel }}{ }^{-1}\right)$. Note that the PSF has a clear asymmetry in the southwest direction of the Airy ring. The two vertical bars correspond to $10 \mathrm{kpc}$.
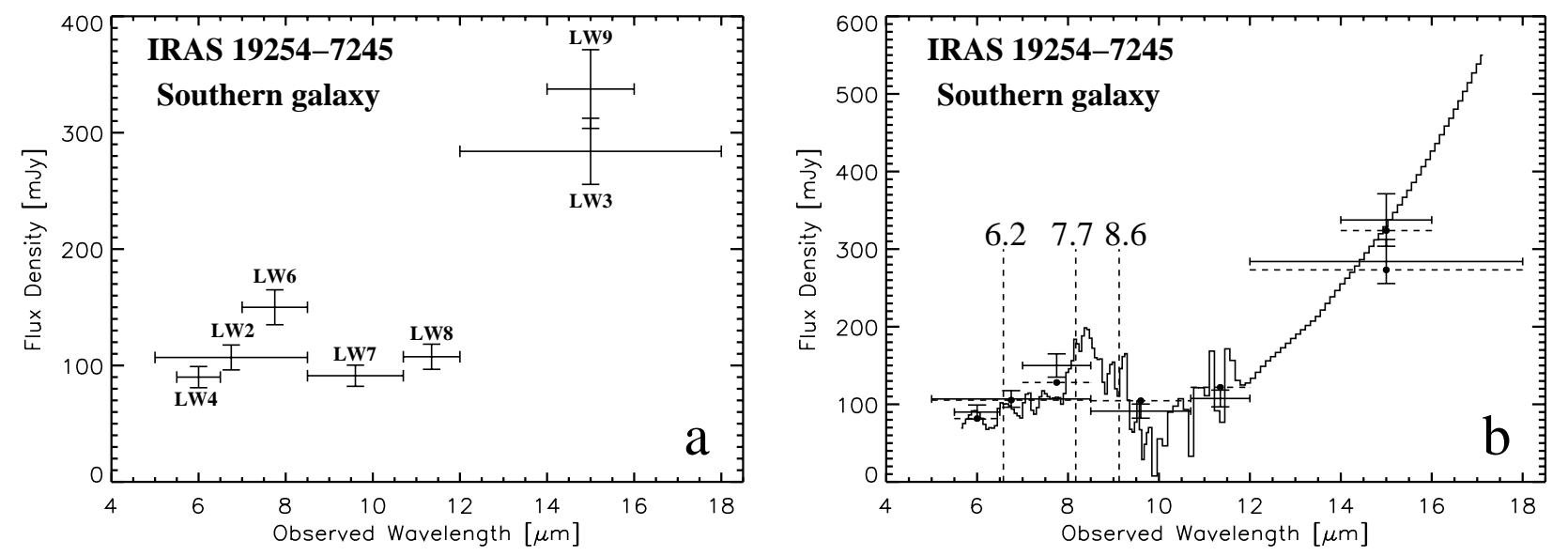

Fig. 2. a) The MIR spectral energy distribution (SED) of the southern component of IRAS 19254-7245 based on the 7 ISOCAM broad band filters. The width of each filter is indicated by a horizontal line and the photometric uncertainties by the vertical lines. b) Same as a) including the ISOPHOT spectrum of the southern component. Since the ISOPHOT beam covered the both galaxies the latter was estimated after subtracting the template MIR spectrum of M 82 for the emission from the northern companion which is, as we see as well in Fig. 3, necessary to explain the high $L W 9 / L W 3$ ratio. The ISOPHOT data end at $\sim 12 \mu \mathrm{m}$, but for instructive purposes we mark the continuum between 12 and $17 \mu \mathrm{m}$ with a power law after having subtracted the weak contribution from the northern galaxy. To visually estimate the quality of the fit we include again for comparison the observed flux in this galaxy presented in the left panel. The positions of the main UIB features, redshifted due to the distance of the galaxy are also marked. The elevated MIR fluxes near $5 \mu \mathrm{m}$ suggest that this galaxy, classified optically as Seyfert 2 , has the typical MIR characteristics of an AGN.

in ionized regions close to young stars (Mirabel et al. 1998) or AGN Laurent et al. (2000). On the contrary, the northern galaxy has strong UIBs $\left(f_{6.7} \mu \mathrm{m} / f_{6} \mu \mathrm{m} \sim 2.5\right)$ and faint thermal emission at $15 \mu \mathrm{m}\left(f_{15 \mu \mathrm{m}} / f_{6.7 \mu \mathrm{m}} \sim 1.2\right)$, which is typical of MIR emission from normal spiral galaxies with cool IRAS colors (Dale et al. 2001; Roussel et al. 2001). Comparison of its broad-band SED with the template SED of a quiescent star forming region within the disk of M 82 (Laurent et al. 2000) illustrates that they are in a fairly good agreement (Fig. 3).
The absolute luminosities presented in Table 5 shows that the MIR emission originating from the southern Seyfert 2 galaxy is by far the strongest in our sample although the most luminous FIR source is IRAS 14348-1447 (see Table 1). One may also note that the flux density near $5 \mu \mathrm{m}$ does not reach zero level but is $\sim 100 \mathrm{mJy}$, suggesting the presence of a hot dust component, which as discussed in the previous section is a clear sign of a hot dusty torus of an AGN (Laurent et al. 2000). Similarly, one can draw the same conclusion by 

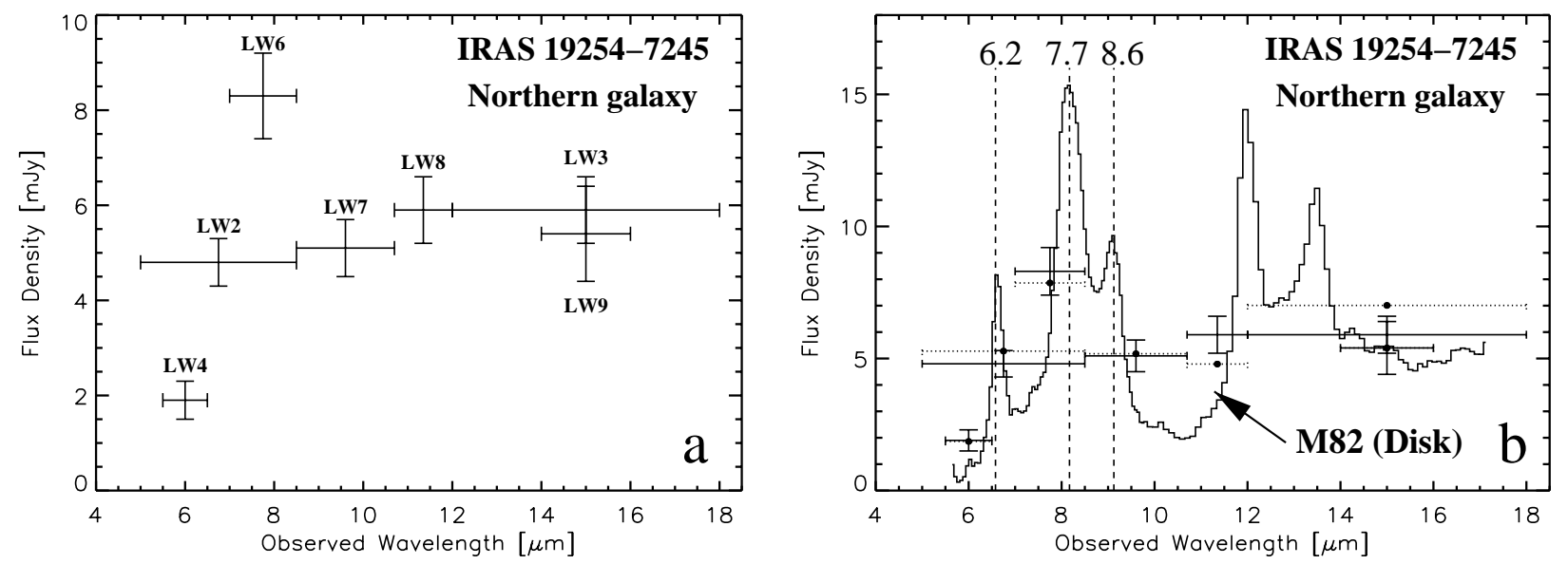

Fig. 3. a) The MIR spectral energy distribution (SED) of the northern component of IRAS 19254-7245 based on the 7 ISOCAM broad band filters. Again the width of each filter is indicated by a horizontal line and the photometric uncertainties by the vertical lines. b) The same SED including a template fit of CVF spectrum (solid line) from a quiescent star forming region in the disk of M 82 . The spectrum of M 82 has been normalized to the flux density sampled by the LW9 filter of IRAS 19254-7245, which traces essentially the VSG component. The dotted lines are the equivalent broad-band filter fluxes of the M 82 template. The small offset between those and the actual measurements of IRAS 19254-7245, indicate that the northern galaxy is dominated in the MIR by starburst activity.

Table 5. Global characteristics. The IR luminosities and the $\mathrm{H}_{2}$ mass are in solar units (see Mirabel et al. 1990).

\begin{tabular}{lccccc}
\hline \hline Target & $L_{L W 2}$ & $L_{L W 3}$ & $\frac{L_{L W 2}}{L^{2}}$ & $\frac{L_{L W 3}}{L_{\mathrm{IR}}}$ & $\frac{L_{\mathrm{IR}}}{M_{\mathrm{H}_{2}}}$ \\
\hline $19254-7245(\mathrm{~S})$ & 53.8 & 42.9 & - & - & - \\
$19254-7245(\mathrm{~N})$ & 1.1 & 0.9 & - & - & - \\
$19254-7245$ & 54.9 & 43.8 & 0.05 & 0.04 & 34.1 \\
& & & & & \\
$23128-5919(\mathrm{~S})$ & 17.7 & 19.2 & - & - & - \\
$23128-5919(\mathrm{~N})$ & 9.6 & 7.5 & - & - & - \\
$23128-5919$ & 27.3 & 26.7 & 0.03 & 0.03 & 70.2 \\
& & & & & \\
$14348-1447(\mathrm{~S})$ & 18.9 & 21.6 & - & - & - \\
$14348-1447(\mathrm{~N})$ & 9.8 & 6.7 & - & - & - \\
$14348-1447$ & 28.7 & 28.3 & 0.02 & 0.02 & 31.0 \\
\hline
\end{tabular}

observing the combination of the $f_{15 \mu \mathrm{m}} / f_{6.7 \mu \mathrm{m}}$ and $f_{6.7 \mu \mathrm{m}} / f_{6 \mu \mathrm{m}}$ flux ratios. In IRAS 19254-7245S, the low $f_{6.7 \mu \mathrm{m}} / f_{6 \mu \mathrm{m}}$ indicates weak UIB emission while $f_{15 \mu \mathrm{m}} / f_{6.7 \mu \mathrm{m}} \sim 2.7$, a value somewhat lower than other well studied starburst galaxies such as Arp $220\left(f_{15 \mu \mathrm{m}} / f_{6.7 \mu \mathrm{m}} \sim 3.9\right.$, Charmandaris et al. 1999b) or the extremely strong starburst region in the Cartwheel $\left(f_{15 \mu \mathrm{m}} / f_{6.7 \mu \mathrm{m}} \sim 5.2\right.$, Charmandaris et al. 1999a). This effect can be understood since the hot continuum produced by an AGN at short MIR wavelengths would cause the flux in the $6-10 \mu \mathrm{m}$ range to increase more relative to the increase observed between $12-16 \mu \mathrm{m}$ and as result it would be added the UIB emission sampled by the $L W 2$ filter.

Could the large difference in the MIR brightness between the north and south component in IRAS 19254-7245 be related to the additional contribution of the AGN? Studies of the dynamical evolution of this system suggest that the starburst time scale is much shorter than the dynamical age of the merger (Mihos \& Bothun 1998). Even though we can not quantify

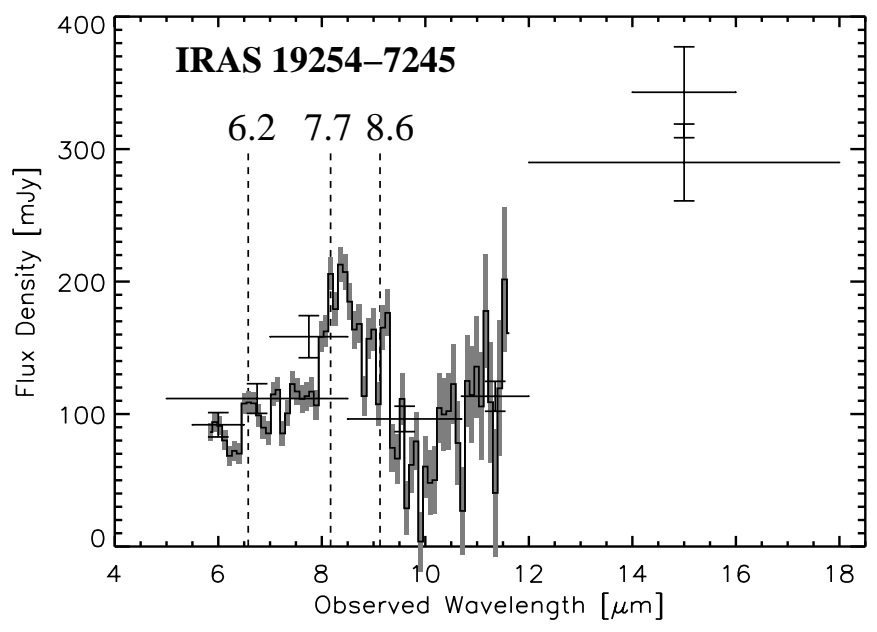

Fig. 4. The integrated MIR SED of IRAS 19254-7245. Our measurements using the 7 ISOCAM broad band filters (solid horizontal lines) are compared to the ISOPHOT-S spectrum. The photometric uncertainties of the ISOPHOT-S are indicated by the hashed zones. The positions of the main UIB features, redshifted due to the distance of the galaxy are again marked.

accurately the fraction of MIR luminosity due to the AGN activity, it appears that the southern component of IRAS 192547245 has reached an AGN dominant phase, however short this may be, after an initial phase of strong starburst activity (see Laurent et al. 2000 and Genzel et al. 1998 for details on the MIR AGN/starburst fraction of this and other galaxies). The MIR properties of the northern nucleus are similar to a normal spiral galaxy which indicates that even if a starburst did occur in it at some point, it has by now subsided and the star formation is progressing in a more quiescent rate.

Finally, we note that the southern galaxy exhibits higher $f_{15 \mu \mathrm{m}} / \mathrm{H} \alpha(\sim 225)$ compared to that in the north $(\sim 85)$. We interpret this effect as a consequence of higher dust concentration and stronger absorption in the southern nucleus since near 

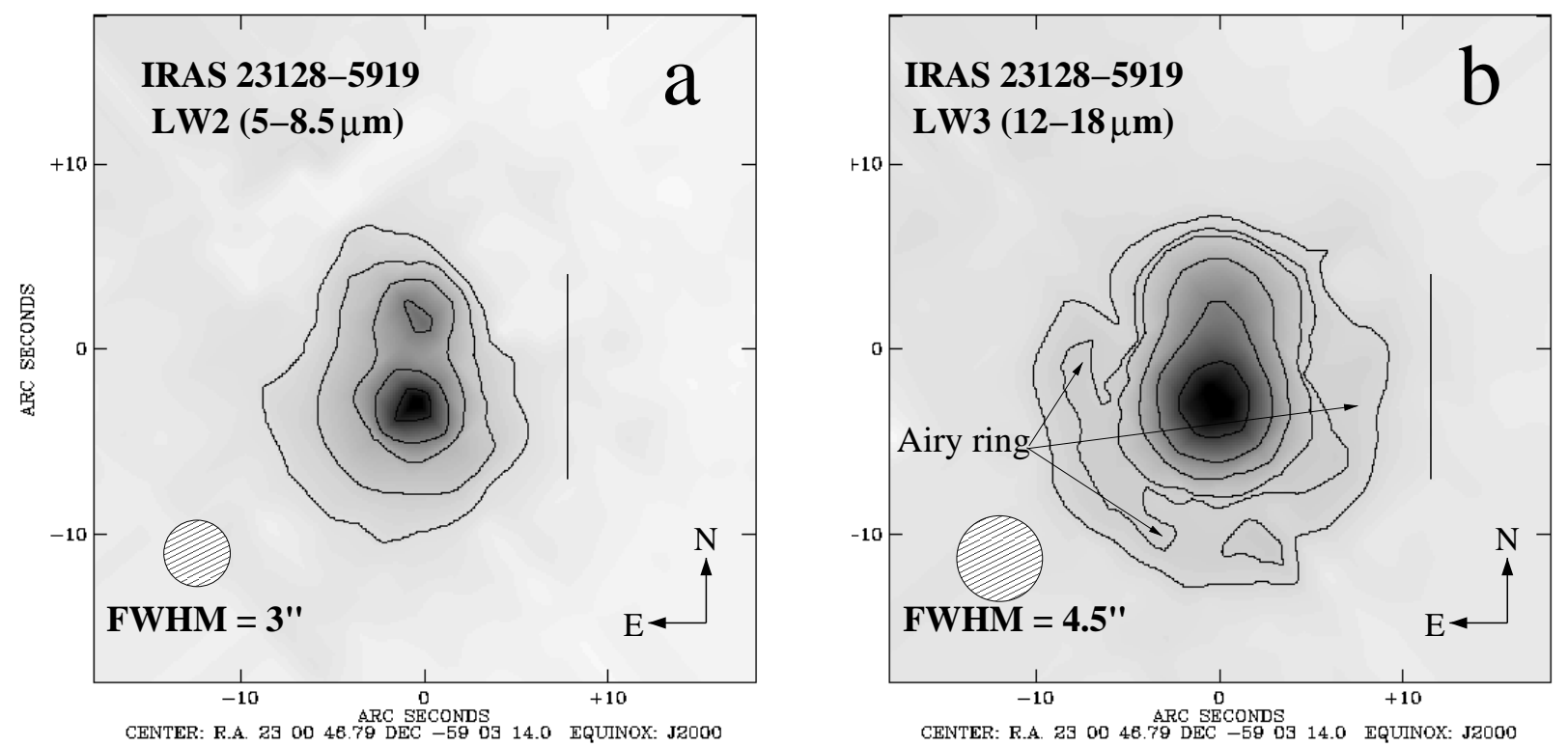

Fig. 5. a) Image of IRAS $23128-5919$ observed with ISOCAM at $6.75 \mu \mathrm{m}$ ( $L W 2$ filter). The contours are $5,10,20,40$ and $80 \sigma$ $\left(\sigma=0.055 \mathrm{mJy} \mathrm{pixel}^{-1}\right)$. b) Image of IRAS 23128-5919 observed with ISOCAM at $15 \mu \mathrm{m}$ ( $L W 3$ filter). The contours are $5,8,10,20,40$ and $80 \sigma\left(\sigma=0.093 \mathrm{mJy}_{\text {pixel }}{ }^{-1}\right)$. Note the asymmetry of the PSF towards the southeast of the Airy ring. The two vertical bars correspond to $10 \mathrm{kpc}$.
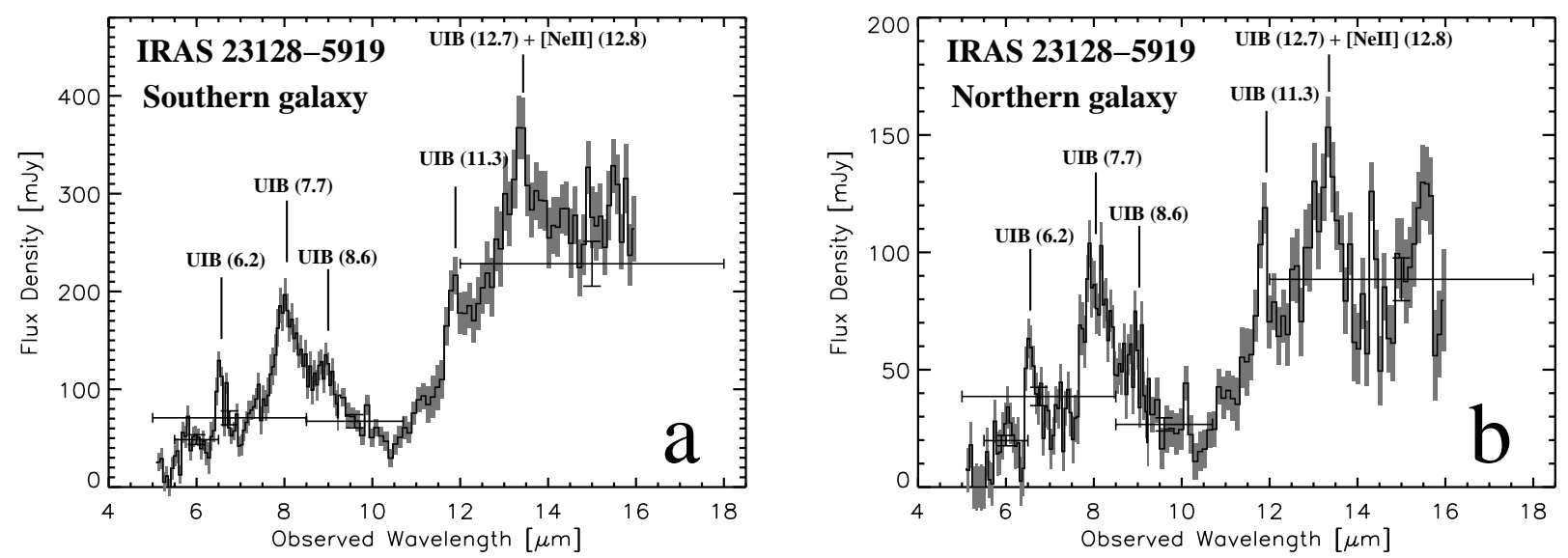

Fig. 6. a) The ISOCAM spectrum of the southern galaxy of IRAS 23128-5919. The flux densities of the 4 ISOCAM broad band filters are superimposed on the CVF spectrum using horizontal lines, the width of which denotes as usual the filter bandpass. The photometric uncertainties of the CVF are indicated by the hashed zones. b) The ISOCAM spectrum of the northern galaxy of IRAS 23128-5919 using the same convention. All prominent UIB features redshifted to the distance of the galaxy are marked.

AGNs high column densities of molecular gas are typically observed. The southern galaxy also has a higher $f_{15 \mu \mathrm{m}} / K$ ratio than that in the north, which has an $f_{15} \mu \mathrm{m} / K$ ratio of a normal spiral galaxy,consistent with its overall MIR spectral features (Table 4).

\subsection{IRAS $23128-5919$}

This system consists of two merging galaxies in a rather late stage of their interaction, the nuclei of which are separated by a projected distance of $4 \mathrm{kpc}\left(5^{\prime \prime}\right)$ (Fig. 5). Two tidal tails $40 \mathrm{kpc}$ stretch in opposite directions (Bergvall \& Johansson 1985; Mihos \& Bothun 1998).

Based on optical studies, the northern galaxy is classified as a starburst, while it is unclear whether the southern one is a Seyfert, a starburst or a LINER (Duc \& Mirabel 1997a). Optical spectroscopy of the southern nucleus shows a relatively high ionization state having emission lines with wings of $\sim 1500 \mathrm{~km} \mathrm{~s}^{-1}$ larger in the blue and extending $\sim 5 \mathrm{kpc}$ out from the nucleus. These emission lines, as well as other WolfRayet features observed, could be caused by supernova winds and turbulent motions associated with the merger (Johansson $\&$ Bergvall 1988). The northern galaxy on the other hand, has narrower emission lines and weaker starburst activity.

In Fig. 6, we present the CVF spectra of each galaxy along with our flux measurements using the four broad-band filters. The integrated MIR SED of the whole system is displayed in Fig. 7, as well as the ISOPHOT-S spectrum which is in good agreement with our data. As in the case of IRAS 19254-7245, 

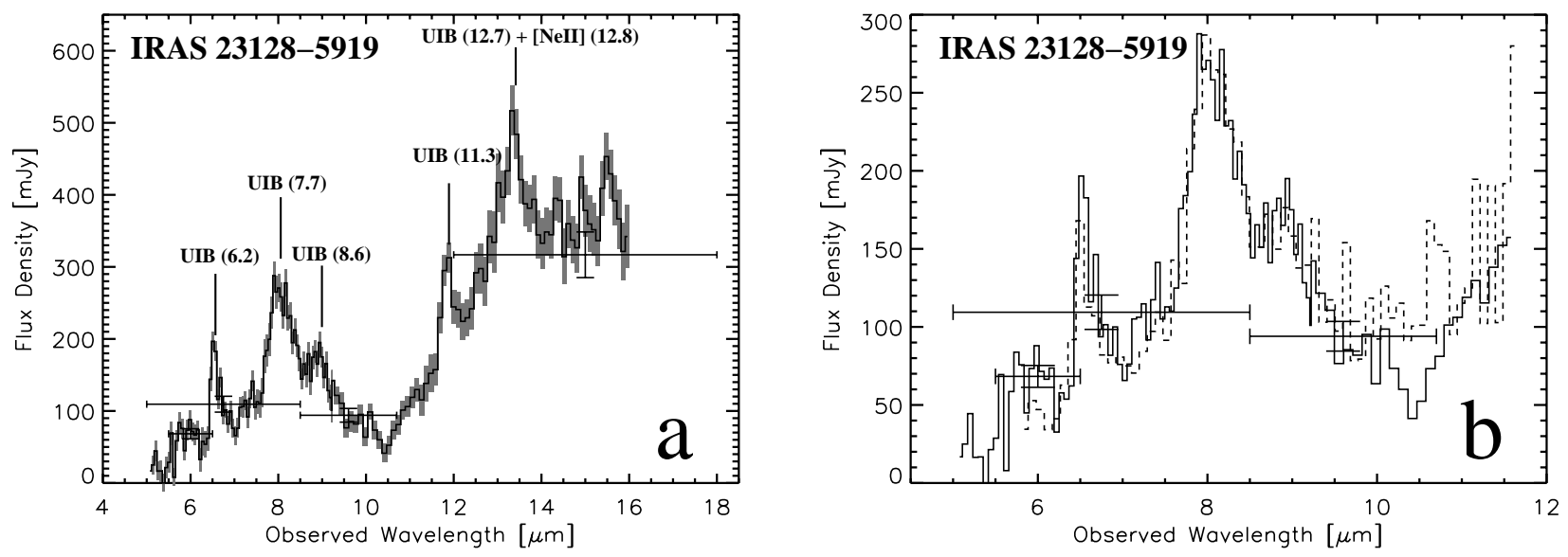

Fig. 7. a) The integrated ISOCAM MIR spectrum of IRAS 23128-5919 using the same conventions as in the previous figures. b) The ISOCAM spectrum of IRAS 23128-5919 (solid line) superimposed on the ISOPHOT-S spectrum (dashed line). Our measurements using the ISOCAM broad band filters are also included.

no MIR emission is seen to be associated with the tidal tails down to our sensitivity limits (see Fig. 5).

We find that approximately $75 \%$ of the MIR flux in IRAS 23128-5919 originates from the southern galaxy. The spectrum reveals that the thermal continuum $(12-16 \mu \mathrm{m})$ is higher in the southern galaxy than that of the north, making the southern galaxy the dominant origin of the MIR emission. Since the SED of both components displays a rising spectrum with prominent UIBs and a weak continuum at 5-6 $\mu \mathrm{m}$, we conclude that the MIR emission in this system is mostly powered by massive star formation. The same conclusion can be reached using the broad-band filter flux ratios for the two galaxies. In the northern more quiescent galaxy of the pair, the MIR activity indicator $f_{15 \mu \mathrm{m} / f_{6.7} \mu \mathrm{m}}(L W 3 / L W 2)$ is 2.6, lower than the value of the southern galaxy $(\sim 3.3)$, while its ratio of $f_{6.7 \mu \mathrm{m}} / f_{6 \mu \mathrm{m}}$ is $\sim 2.0$, higher than that of the southern galaxy which has an $f_{6.7 \mu \mathrm{m}} / f_{6 \mu \mathrm{m}} \sim 1.5$. Following similar reasoning as for the southern component of the Superantennae, these results can be interpreted as an increase in the density of $\mathrm{H}$ II regions of the southern component, relative to the density of the photo-dissociation regions. Further comparisons of the properties of this galaxy to IRAS 19254-7245 (see Table 5) show that its ratio of $L_{L W 3} / L_{\mathrm{IR}} \sim 0.03$ is smaller despite is high $L_{\mathrm{IR}}\left(L_{\odot}\right) / M_{\mathrm{H}_{2}}\left(M_{\odot}\right)$ of $\sim 70$. This indicates that even though IRAS 23128-5919 is more efficient in consuming the molecular gas, its radiation field is not sufficient to heat the large amount of dust at similarly high temperatures as does the AGN in the Superantennae. The data presented in Table 4 also indicate that the southern galaxy of the pair emits more MIR flux relatively to its stellar emission $\left(f_{15 \mu \mathrm{m}} / K \sim 284\right)$ and is apparently more obscured by dust $\left(f_{15 \mu \mathrm{m}} / \mathrm{H} \alpha \sim 85\right)$.

In conclusion, the more luminous galaxy is clearly undergoing a stronger star formation phase than its northern companion. The global MIR characteristics of this system are in agreement with the assertion that a starburst is the dominant heating mechanism for the dust and no evidence of an AGN contributing to the ISOCAM wavelength range are present.

\subsection{IRAS 14348-1447}

IRAS 14348-1447 is the most distant object in the IRAS Bright Galaxy Sample with a redshift of 0.08 (Soifer et al. 1987). This system, shown in Fig. 8, consists of two galaxies separated by a projected distance of $6 \mathrm{kpc}\left(4^{\prime \prime}\right)$ with a tail extending to more than $10 \mathrm{kpc}$ away from the northern nucleus (Melnick \& Mirabel 1990). Strong $\mathrm{H}_{2}$ emission, mainly triggered by shocks in molecular clouds, has been detected (Geballe 1988; Nakajima et al. 1991). The presence of large quantities of shocked molecular hydrogen is consistent with the detection of $6 \times 10^{10} M_{\odot}$ of molecular gas in this system which makes it the most $\mathrm{H}_{2}$-rich in the ultraluminous galaxy sample (Sanders et al. 1991). The large quantities of cold dust, inferred using the usual gas to dust conversion, lead us to believe that the reddening seen in both galaxies is a consequence of strong absorption and not due to an intrinsically old stellar population (Carico et al. 1990a).

Based on near-infrared spectroscopic observations in $\mathrm{Pa} \alpha$ and $\mathrm{H}_{2}$ lines, the nucleus of the southern galaxy has been classified as a Seyfert 1.5 and the northern one as a Seyfert 2 (Nakajima et al. 1991), while their optical line features are similar to those of LINERs (Veilleux et al. 1995) or Seyfert 2 galaxies (Sanders et al. 1988).

Due to its relatively weak MIR emission this source was only observed with the two ISOCAM broad band filters $L W 2$ and $L W 3$ (Table 2). As in the other galaxies in this sample, MIR emission is detected only from the circumnuclear regions. We estimate that $\sim 75 \%$ of the MIR flux seen in both filters originates from the southern galaxy, which is also the more active one in the optical. Interestingly, this roughly scales with the fraction of the $\mathrm{CO}$ emission from the two components (Evans et al. 2000). The southern galaxy exhibits the higher hot dust component traced by $15 \mu \mathrm{m}(L W 3)$ relative to the UIB emission at $7 \mu \mathrm{m}(L W 2)$. Using the $L W 3 / L W 2$ ratio to trace the MIR activity in this system we find that $f_{15 \mu \mathrm{m}} / f_{6.7 \mu \mathrm{m}} \sim 3.4$ in the southern galaxy and $f_{15 \mu \mathrm{m}} / f_{6.7 \mu \mathrm{m}} \sim 2.0$ in the northern one. Since we only have one MIR color, we can not comment on the MIR contribution the AGN. Nevertheless, the low 

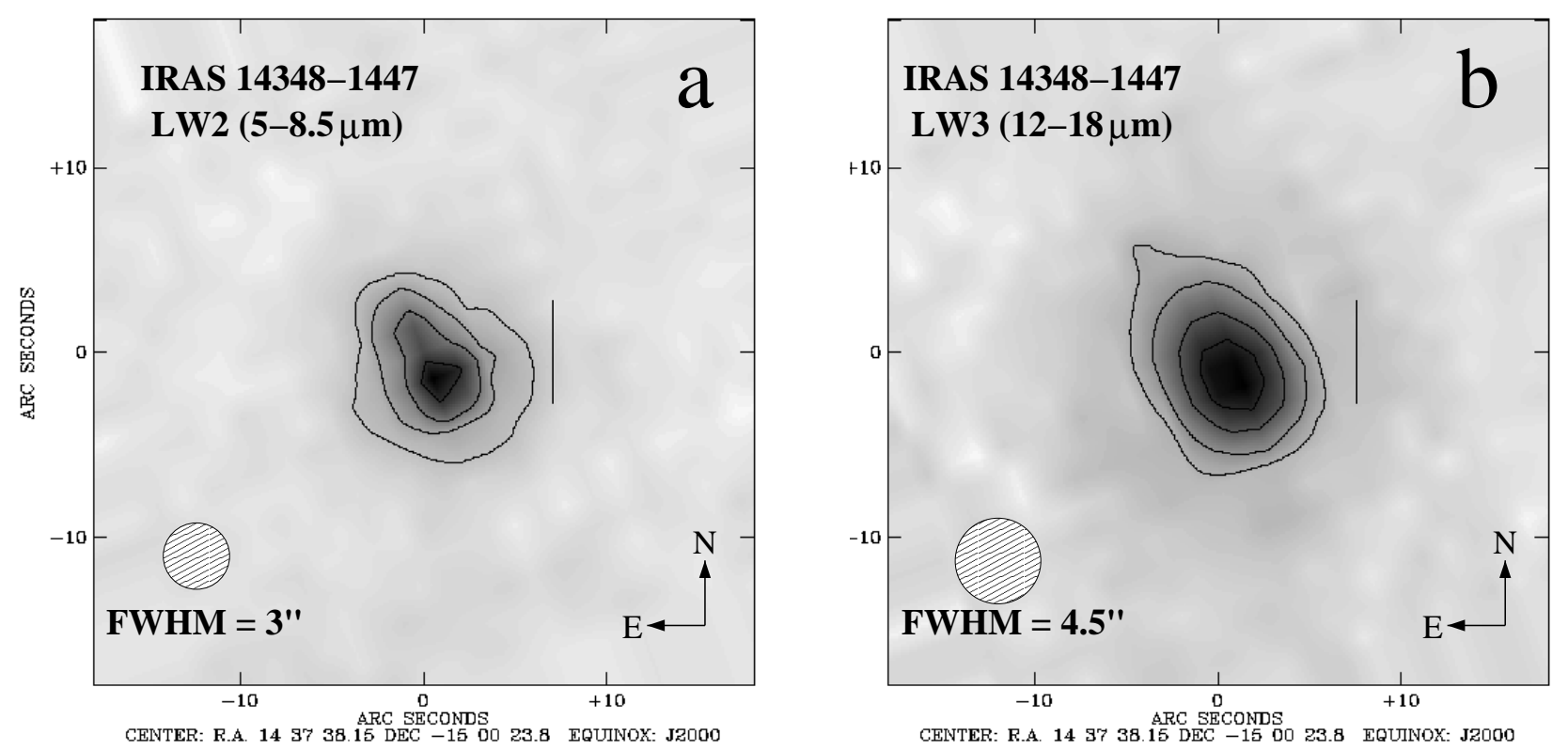

Fig. 8. a) Image of IRAS $14348-1447$ observed with ISOCAM at $6.75 \mu \mathrm{m}$ ( $L W 2$ filter). The contours are $5,10,20,40$ and $80 \sigma$ $\left(\sigma=0.048 \mathrm{mJy}\right.$ pixel $\left.^{-1}\right)$. b) Image of IRAS 14348-1447 observed with ISOCAM at $15 \mu \mathrm{m}$ ( $L W 3$ filter). The contours are 5, 10, 20 and $30 \sigma\left(\sigma=0.073 \mathrm{mJy}\right.$ pixel $\left.^{-1}\right)$. The two vertical bars correspond to $10 \mathrm{kpc}$.

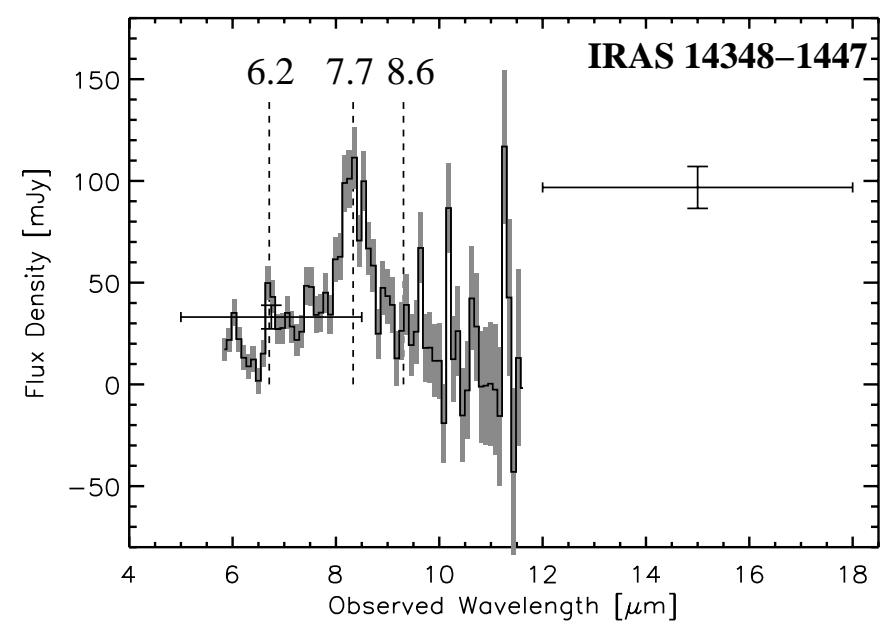

Fig. 9. The ISOPHOT-S spectrum of IRAS 14348-1447 including the measurements of the two ISOCAM filters. The uncertainties of the ISOPHOT-S spectrum are indicated by the hashed zones.

integrated $L_{\mathrm{LW} 3} / L_{\mathrm{IR}}$ of IRAS $14348-1447(\sim 0.02)$ would be consistent with a negligible AGN contribution in the MIR (Table 5) while the high dust obscuration suggested by the increased $f_{15 \mu \mathrm{m}} / \mathrm{H} \alpha \sim 333$ is consistent with its large molecular gas content (Mirabel et al. 1990).

Evidence that the starburst activity is the main heating mechanism can also be seen in Fig. 9 using the MIR spectrum of the whole system obtained with ISOPHOT. This spectrum reveals strong UIBs $\left(f_{6.7 \mu \mathrm{m}} / f_{6 \mu \mathrm{m}} \sim 2.2\right.$, see Table 3$)$ likely caused by a starburst with only a weak contamination by an AGN (to the 25\% level, see Genzel et al. 1998; Lutz et al. 1998).

\section{Discussion and concluding remarks}

A wealth of observational data available has shown that ULIRGs have high concentrations of gas and dust in their nuclei, sufficient to account for most of their observed infrared luminosity (see Sanders \& Mirabel 1996 for a review). Whether the energy source of ULIRGs is a dust enshrouded AGN or a starburst still remains an open issue. However, recent indirect evidence is beginning to favour the existence of bright extremely red point-like sources in the nuclear regions of ULIRGs. More specifically near-infrared observations of luminous infrared galaxies have shown that their flux at $2.2 \mu \mathrm{m}$ is more concentrated towards the center than at $1.3 \mu \mathrm{m}$ (Carico et al. 1990b; Scoville et al. 2000). Furthermore, recent high resolution MIR observations using Keck of a sample of ULIRGs reveal compact sub-arcsecond sources (with linear scales of $\sim 100-300 \mathrm{pc}$ ) which contain $30 \%$ to $100 \%$ of the observed MIR energy of these galaxies (Soifer et al. 2000). This contrasts with the LIRGs $\left(10^{11} L_{\odot} \leq L_{\mathrm{IR}} \leq 10^{12} L_{\odot}\right)$, in which the infrared energy seems to be generated over somewhat larger scales ( 100 pc-1 kpc, Soifer et al. 2001) and sometimes can be found in extra-nuclear regions associated with the physical interaction of merging pairs of galaxies. Furthermore, there are galaxies such as VV 114 where it has even been found that a substantial fraction of the MIR flux originates from an extended component of hot dust emission spread over several kpc scales (Soifer et al. 2001; Le Floc'h et al. 2002). ULIRGs are thus not simply a scaled-up version of LIRGs and require further dynamical compression of the molecular gas responsible for the IR luminosity within very compact regions. A plausible mechanism would be one where the shocks and tidal forces of the interaction first lead to star formation over galactic scales, leading to IR luminosities up to a few $10^{11} L_{\odot}$. Subsequently, gravitational instabilities and the formation of a bar, strip the gas 
of its angular momentum, funneling large quantities towards the nuclear regions of galaxies, which can feed circumnuclear starbursts or AGNs and trigger the ultraluminous phase in the infrared (Combes 2001).

Even though the above scenario is appealing, given the high extinction in the nuclei of ULIRGs, the limited atmospheric transmission in the MIR windows, and the limited sensitivity of ground-based instruments, questions related to the direct probing of the nuclear activity such as "does all MIR emission from those systems originate from the nuclei?" and if not "what are the spectral properties of any extended component?" still remain unanswered.

This is where the superb sensitivity of space instruments, such as ISO, is essential. We have found that in the ULIRGs studied here more than $~ 95 \%$ of the MIR emission seen by IRAS is confined within a few arcsecs of their central region. Obviously the relatively large pixel size of the ISOCAM detector places limitations in interpreting these findings. However, deconvolution tests of the central point source in each galaxy suggest that the corresponding nuclei are resolved and the physical diameter of the emitting region is contained within 1 to $2 \mathrm{kpc}$. Moreover, with the exception of the Superantennae where the MIR spectrum is dominated by the emission arising from the AGN of the southern galaxy, the bulk of the IR luminosity of IRAS $23128-5919$ and IRAS 14348-1447 is powered by massive star formation. The fact that starbursts can dominate the MIR emission in galaxies with IR luminosities as high $\sim 10^{12} L_{\odot}$ had already been demonstrated in other ISOCAMCVF (Tran et al. 2001) and ISO-SWS (Genzel et al. 1998) observations of ULIRGs, and is supported by our results. Given that an active nucleus appears to be always present in the most energetic objects of the local Universe (Lutz et al. 1998), our MIR data favor a luminosity threshold for the transition between starburst- and AGN-dominated galaxies which is higher than the IR luminosity of the galaxies in our sample. This is in agreement with the results of Tran et al. (2001) who proposed that this transition takes place at $L_{\mathrm{IR}} \sim 10^{12.5} L_{\odot}$ and also found individual starbursts up to $10^{12.65} L_{\odot}$. Our data also indicate is that such starbursts can be confined to the very central nuclear regions which may have important consequences in the probing how the instabilities fuel the inner regions of galaxies (e.g. Combes 2001), as well as determining the nature of high redshift dusty sources (e.g. Ivison et al. 2000).

Another striking feature revealed in our observations is that in all three cases one galaxy seems to dominate the MIR energy output of the system by more than $75 \%$. Could this be a record of the initial distribution of the amount of molecular gas available in each merging progenitor or could this suggest that in the later stages of interaction, the gas finally merges towards one component? If the latter were true one would expect that a sufficiently large quantity of gas could trigger and fuel both circumnuclear star forming activity and AGN-type activity at the core of a single object. This is evident in the southern galaxy of IRAS 19254-7245 which harbors an active nucleus as well as numerous massive star forming regions. As we mentioned in the introduction though the presence of a Seyfert nucleus is correlated with a MIR flux increase relative to the FIR luminosity of the entire galaxy, which is what one can actually derive from our observations when we compare the Superantennae with IRAS 14348-1447. IRAS 14348-1447 has indeed a much higher total IR luminosity despite its MIR flux being lower than that of the southern source of IRAS 19254-7245. Furthermore, using the $f_{15 \mu \mathrm{m}} / \mathrm{H} \alpha$ and $f_{15 \mu \mathrm{m}} / K$ ratios as probes of dust absorption and hot dust emission normalized to the mass of the galaxy, we find that in each interacting system it is always the most active galaxy of the system that exhibits the higher ratios. In each system, the most luminous galaxy contains a larger amount of molecular gas leading to the triggering/feeding of the starburst activity and/or an active nucleus.

Finally, we wish to stress once more that because of the limited spatial resolution in studying such distant sources, the diagnostics we have used in this paper address only the integrated MIR emission of each galaxy. Our difficulties to identify whether an active nucleus is solely responsible for the increase in the MIR luminosity relative to the FIR emission will not be resolved unless we can either clearly map the extent of the emitting region or obtain MIR spectra using very narrow slits. The upcoming launch of SIRTF which, despite the fact it has comparable spatial resolution to ISO, is equipped with a new generation of detectors of smaller pixel size, and in particular the use of its infrared spectrograph will help us improve upon our current results and provide conclusive answers to the issues which still remain unresolved to date.

Acknowledgements. We wish to thank P.-A Duc for providing his $K$ band images of IRAS 19254-7245 and IRAS 23128-5919 as well as D. Rigopoulou for providing the ISOPHOT data. We greatly appreciated the comments of the referee which helped us improve the manuscript. VC would like to acknowledge the partial support of JPL contract 960803 .

\section{References}

Barvainis, R. 1987, ApJ, 320, 537

Bergvall, N., \& Johansson, L. 1985, A\&A, 149, 475

Borne, K. D., Bushouse, H., Colina, L., et al. 1999, Ap\&SS, 266, 137

Boselli, A., Lequeux, J., Contursi, A., et al. 1997, A\&A, 324, L13

Bryant, P. M., \& Scoville, N. Z. 1999, AJ, 117, 2632

Carico, D. P., Graham, J. R., Matthews, K., et al. 1990a, ApJ, 349, L39

Carico, D. P., Sanders, D. B., Soifer, B. T., Matthews, K., \& Neugebauer, G. 1990b, AJ, 100, 70

Cesarsky, C. J., Abergel, A., Agnese, P., et al. 1996a, A\&A, 315, L32

Cesarsky, D., Lequeux, J., Abergel, A., et al. 1996b, A\&A, 315, L305

Charmandaris, V., Laurent, O., Mirabel, I. F., et al. 1999a, A\&A, 341, 69

Charmandaris, V., Laurent, O., Mirabel, I. F., et al. 1999b, Ap\&SS, 266, 99

Charmandaris, V., Le Floc'h, E., Laurent, O., et al. 2002, ApJ, in preparation

Clavel, J., Schulz, B., Altieri, B., et al. 2000, A\&A, 357, 839

Colina, L., Lipari, S., \& Macchetto, F. 1991, ApJ, 379, 113

Combes, F. 2001, Fueling the AGN. In Lectures on the StarburstAGN Connection, INAOE, ed. D. Kunth, \& I. Aretxaga [astro-ph/0010570]

Condon, J. J., Huang, Z.-P., Yin, Q. F., \& Thuan, T. X. 1991, ApJ, 378, 65

Coulais, A., \& Abergel, A. 2000, A\&AS, 141, 533

de Grijp, M. H. K., Miley, G. K., Lub, J., \& de Jong, T. 1985, Nature, 314, 240 
Dale, D. A., Helou, G., Contursi, A., Silbermann, N. A., \& Kolhatkar, S. 2001, ApJ, 549, 215

Duc, P. A., \& Mirabel, I. F. 1997a, The Messenger, 89, 14

Duc, P. A., Mirabel, I. F., \& Maza, J. 1997b, A\&AS, 124, 533

Dudley, C. C. 1999, MNRAS, 307, 553

Evans, A. S., Surace, J. A., \& Mazzarella, J. M. 2000, ApJ, 529, L88

Geballe, T. R. 1988, MNRAS, 234, 1P

Genzel, R., Lutz, D., Sturm, E., et al. 1998, ApJ, 498, 579

Helou, G., Malhotra, S., Hollenbach, D. J., Dale, D. A., \& Contursi, A. 2001, ApJ, 549, 215

Houck, J. R., Soifer, B. T., Neugebauer, G., et al. 1984, ApJ, 278, L63 Imanishi, M., \& Dudley, C. C. 2000, ApJ, 545, 701

Ivison, R. J., Smail, I., Barger, A. J., et al. 2000, MNRAS, 315, 209

Johansson, L., \& Bergvall, N. 1988, A\&A, 192, 81

Joseph, R. D. 1999, Ap\&SS, 266, 321

Kessler, M. F., Steinz, J. A., Anderegg, M. E., et al. 1996, A\&A, 315, L27

Laurent, O. 1999a, Ph.D. Thesis, University of Paris XI, France

Laurent, O., Mirabel, I.F., Charmandaris, V., et al. 1999b, in XIXth Moriond Astrophysics Meeting. Building the Galaxies: From the Primordial Universe to the Present, 79 [astro-ph/0005377]

Laurent, O., Mirabel, I. F., Charmandaris, V., et al. 2000, A\&A, 359, 887

Le Floc'h, E., Mirabel, I. F., Laurent, O., et al. 2001, A\&A, 367, 487

Le Floc'h, E., Charmandaris, V., Laurent, O., et al. 2002, A\&A, 391, 417

Leger, A., D’Hendecourt, L., Boissel, P., \& Desert, F. X. 1989, A\&A, 213,351

Lutz, D., Spoon, H. W. W., Rigopoulou, D., Moorwood, A. F. M., \& Genzel, R. 1998, ApJ, 505, L103

Madden, S. C., Vigroux, L., \& Sauvage, M. 1997, in Extragalactic Astronomy in the Infrared, ed. G. A. Mamon, T. X. Thuan, \& J. Tran Thanh Van, 229

Mathis, J. S. 1990, ARA\&A, 28, 37

Melnick, J., \& Mirabel, I. F. 1990, A\&A, 231, L19

Mihos, J. C., \& Bothun, G. D. 1998, ApJ, 500, 619

Mirabel, I. F., Booth, R. S., Johansson, L. E. B., Garay, G., \& Sanders, D. B. $1990, A \& A, 236,327$
Mirabel, I. F., Lutz, D., \& Maza, J. 1991, A\&A, 243, 367

Mirabel, I. F., Vigroux, L., Charmandaris, V., et al. 1998, A\&A, 333, L1

Nakajima, T., Kawara, K., Nishida, M., \& Gregory, B. 1991, ApJ, 373, 452

Pier, E. A., \& Krolik, J. H. 1992, ApJ, 401, 99

Richstone, D., Ajhar, E. A., Bender, R., et al. 1998, Nature, 395, A14

Rigopoulou, D., Spoon, H. W. W., Genzel, R., et al. 1999, AJ, 118, 2625

Roche, P. F., Aitken, D. K., Smith, C. H., \& Ward, M. J. 1991, MNRAS, 248, 606

Roussel, H., Sauvage, M., Vigroux, L., \& Bosma, A. 2001, A\&A, 372, 427

Sanders, D. B., \& Mirabel, I. F. 1985, ApJ, 298, 31

Sanders, D. B., Scoville, N. Z., Young, J. S., et al. ApJ, 305, 45L

Sanders, D. B., Soifer, B. T., Elias, J. H., et al. 1988, ApJ, 325, 74

Sanders, D. B., Scoville, N. Z., \& Soifer, B. T. 1991, ApJ, 370, 158

Sanders, D. B., \& Mirabel, I. F. 1996, ARA\&A, 34, 749

Sanders, D. B. 1999, Ap\&SS, 266, 331

Sauvage, M., Blommaert, J., Boulanger, F., et al. 1996, A\&A, 315, L89

Scoville, N. Z., Evans, A. S., Thompson, R., et al. 2000, AJ, 119, 991

Soifer, B. T., Sanders, D. B., Madore, B. F., et al. 1987, ApJ, 320, 238

Soifer, B. T., Boehmer, L., Neugebauer, G., \& Sanders, D. B. 1989, AJ, 98, 766

Soifer, B. T., Neugebauer, G., Matthews, K., et al. 2000, AJ, 119, 509

Soifer, B. T., Neugebauer, G., Matthews, K., et al. 2001, AJ, 122, 1213

Starck, J. L., Siebenmorgen, R., \& Gredel, R. 1997, ApJ, 482, 1011

Starck, J. L., Abergel, A., Aussel, H., et al. 1999, A\&AS, 134, 135

Wild, W., Harris, A. I., Eckart, A., et al. 1992, A\&A, 265, 447

Tran, Q. D., Lutz, D., Genzel, R., et al. 2001, ApJ, 552, 527

Vanzi, L., Bagnulo, S., Le Floc'h, E., et al. 2002, A\&A, 386, 464

Veilleux, S., Kim, D.-C., Sanders, D. B., Mazzarella, J. M., \& Soifer, B. T. 1995, ApJS, 98, 171

Verstraete, L., Puget, J.-L., Falgarone, E., et al. 1996, A\&A, 315, L337

Vigroux, L., Charmandaris, V., Gallais, P., et al. 1999, in The Universe as Seen by ISO, ESA SP-427, 805 